\title{
Poisoning with amitraz, an overlooked pesticide, in an adolescent boy in Kenya
}

\author{
Serah Kajuju Ngugi
}

\begin{abstract}
Introduction: Amitraz is a formamidine chemical compound used worldwide as an ectoparasiticide and an agricultural pesticide. Despite its widespread use, very few cases of human poisoning have been described leading to low awareness among health care providers. Some of its effects on humans may mimic organophosphate poisoning. As a result, cases of amitraz intoxication are not recognized and are therefore incorrectly treated as the much more commonly recognized organophosphate poisoning. There is currently no known antidote for amitraz poisoning and the management is mainly supportive and symptomatic. Case Report: We report a case of a 12-year-old boy who presented to the emergency department with depressed level of consciousness, miosis, bradycardia and hypothermia following deliberate ingestion of a pesticide. Baseline laboratory studies were notable of hyperglycemia and glycosuria but no ketonuria. His past medical history was unremarkable with no history of diabetes mellitus or drug abuse. The poisoning was initially incorrectly diagnosed as organophosphate intoxication but later changed to amitraz poisoning. He was successfully managed supportively with oxygen supplementation, airway management, intravenous fluids as well as close monitoring.
\end{abstract}

Serah Kajuju Ngugi ${ }^{1,2}$

Affiliations: ${ }^{1}$ MBchB, MMed (Paediatrics and Child Health) County Paediatrician, Ministry of Health, Nyandarua County, Kenya; ${ }^{2}$ Consultant Paediatrician, Department of Paediatrics and Child Health, North Kinangop Catholic Hospital, Nyandarua County, Kenya.

Corresponding Author: Serah Kajuju Ngugi, P.O. Box 34392,

Nairobi,Kenya, 00100; Email: mskajuju@yahoo.com

Received: 11 November 2015

Accepted: 05 February 2016

Published: 01 April 2016
Conclusion: This case report highlights the importance of considering other toxic agents/ chemicals other than organophosphate in patients with poisoning. It also highlights the lifethreatening presentation in amitraz poisoning, as well as the excellent prognosis with early recognition and supportive management.

Keywords: Amitraz, Organophosphate, Pesticides, Poisoning

\section{How to cite this article}

Ngugi SK. Poisoning with amitraz, an overlooked pesticide, in an adolescent boy in Kenya. Case Rep Int 2016;5:9-12.

Article ID: 100022CRINTSN2O16

$* * * * * * * * *$

doi:10.5348/crint-2016-22-CR-3

\section{INTRODUCTION}

Amitraz is a chemical belonging to the formamidine class of compounds. It has acaricide and insecticide properties and is used to control ticks in cattle, sheep, goats and dogs as well as pests in crops [1]. Commercial formulations of amitraz generally contain 12.5-20\% of the drug in organic solvents, especially xylene, an aromatic hydrocarbon [2].

When humans are exposed to amitraz, the symptoms and signs result from both xylene and amitraz [2]. Amitraz acts centrally by activation of presynaptic $\alpha_{2}-$ adrenoceptors causing inhibition of noradrenaline release resulting in suppression of the outflow of sympathetic nervous system activity from the central nervous system (CNS) [1]. This may cause numerous symptoms varying from CNS depression (drowsiness, 
coma, and convulsions), to miosis, or, rarely, mydriasis, respiratory depression, bradycardia, hypotension, hypothermia, hyperglycemia, polyuria, vomiting, decreased gastrointestinal motility, and intestinal distension [1-4]. Xylene on the other hand may cause acute toxic signs such as: CNS depression, ataxia, impaired motor coordination, nystagmus, stupor, coma, and episodes of neuroexcitability [5].

Despite the widespread use of amitraz as an agroveterinary pesticide, human intoxication in Kenya and Africa in general is under reported. To date only two published studies describe this phenomenon in Africa $[1,6]$. Low awareness among the health care providers and the similarities in some of the symptoms to those of organophosphate poisoning, could result in incorrect diagnosis and therefore account for the under reporting of amitraz poisoning. Poisoning in children is most often accidental $[1,3,4]$. This is thought to be first case in Africa describing intentional poisoning in a child below 13 years of age. This case report will therefore contribute to the limited data available and help in increasing awareness regarding this poison, thereby allowing timely recognition and appropriate treatment.

\section{CASE REPORT}

A 12-year-old boy presented to the North Kinangop Catholic Hospital in Nyandarua County, approximately six hours after ingesting an unknown amount of a pesticide, following a disagreement with his mother over studies. He informed his elder sister approximately 20 minutes after consumption and was immediately given some milk after which he vomited. On examination at admission, the child had depressed level of consciousness with a Glasgow coma scale (GCS) of 8/15 and had an abnormal body odor. His pupils were constricted and not reacting to light. He had an axillary temperature of $35^{\circ} \mathrm{C}$, heart rate of 50 beats per minute, blood pressure of $100 / 60 \mathrm{mmHg}$ and a respiratory rate of 14 breaths/ minute. Oxygen saturation at room air was $85 \%$. He had no excessive salivation, sweating or chest congestion. The other examinations were unremarkable.

In view of the bradycardia, miosis and depressed level of consciousness; a presumed diagnosis of organophosphate poisoning was made. An oral airway was introduced and oxygen administered via face mask with improvement in oxygen saturation. He was given an intravenous bolus of atropine at $0.02 \mathrm{mg} / \mathrm{kg}$ which was repeated every 10 minutes and later 2 hourly as the heart rate improved. A nasogastric tube was inserted and gastric lavage done. The gastric contents smelt of a chemical. Warmed intravenous normal saline was commenced and child kept warm.

Baseline laboratory studies done included a random blood sugar, complete blood count, serum urea, electrolytes, creatinine and urine dipstick analysis. The results were notable of a hyperglycemia (random blood sugar $24 \mathrm{mmol} / \mathrm{l}$ ) and glycosuria (2+) but no ketonuria. Other results were as follows; blood urea nitrogen of $6 \mathrm{mmol} / \mathrm{l}$, creatinine $45 \mu \mathrm{mol} / \mathrm{l}$, sodium $136 \mathrm{mEq} / \mathrm{L}$, potassium $3.8 \mathrm{mEq} / \mathrm{L}$. In the complete blood count, the hemoglobin, white blood cells and the platelet counts were $13.5 \mathrm{~g} / \mathrm{dl}, 7000 / \mathrm{mm}^{3}$, and $160000 / \mathrm{mm}^{3}$, respectively. His medical history was unremarkable with no history of diabetes mellitus or drug abuse.

A few hours after admission, his mother revealed that he had consumed Mostraz ${ }^{\circledR}$ (Amitraz), a chemical that she had procured to spray on her cattle to control ticks. The diagnosis was therefore changed to amitraz poisoning and supportive management continued. Within 12 hours of admission, the level of consciousness had gradually improved, blood sugar was decreasing and he was normothermic. By the second day of admission, the heart rate and blood sugar had normalized and the GCS had improved to $14 / 15$ but the boy was noted to be disoriented and had visual hallucinations. By the third day, the sensorium had returned to normal, pupils were now normal in size and reacting adequately to light and he had become ambulant. He was discharged on the fifth day after he and his parents underwent counseling.

\section{DISCUSSION}

Acute poisoning involving pesticides in children is most often accidental. However, in recent decades, there has been a steady increase in self-poisoning among adolescents in suicidal attempts. The attempted deliberate self-harm with amitraz in this adolescent is consistent with a similar case reported in India [7].

Amitraz is used worldwide as an agro-veterinary pesticide and is marketed under many trade names, including taktic, ectodex, mostraz, triatix, metaban, mitac, acadrex, kenaz, and atarac [8]. It is usually supplied as a highly concentrated aqueous solution which has to be adequately diluted before applying on animals or crops. Amitraz can cause poisoning in animals and humans via oral, dermal or inhalation routes [2].

In the first and only other published case of amitraz poisoning in Kenya, Mwita [6] indicated that in Kenya and most of Africa, amitraz is marketed mainly as Triatix ${ }^{\circledR}$. Our patient however had ingested Mostraz $囚$. This shows that amitraz could be available in the local market under different other trade names and therefore it is important for health care providers to try and establish the generic names of the poisons patients report to have consumed.

Despite the widespread use of amitraz in Kenya, many health care providers are unfamiliar of it and therefore of the clinical presentation of patients with amitraz intoxication. The presence of bradycardia, impaired consciousness, respiratory depression, and in some patients, miosis in amitraz poisoning can lead to erroneous diagnosis of organophosphate poisoning. This was the case in our patient as well as that reported by Mwita [6]. 
The presentation of central nervous system depression, miosis, bradycardia, hypothermia, hyperglycemia and glycosuria in our patients were similar to those described elsewhere in other studies [1-4, 6]. Hallucinations and disorientation observed in this patient have also been described and could be due to neuroexcitation caused by xylene, a solvent used in amitraz [6].

The principal clinical effects of amitraz poisoning are related to its stimulation of presynaptic $\alpha_{2}$-adrenoceptors and include CNS depression, bradycardia, respiratory failure, hypotension, miosis (at low doses) or mydriasis (at higher doses) and hypothermia [5]. In some cases, miosis has been observed early in the intoxication followed by progression to mydriasis suggesting that the presynaptic effect is dominant in the early phase and the postsynaptic effect in the late phase of the intoxication [9]. CNS depression, pupillary light reflex and respiratory failure are thought to be dose dependent. Amitraz has antipyretic and anti-inflammatory activity in vivo, and also has been shown to inhibit prostaglandin $\mathrm{E}_{2}$ synthesis [4]. This could have been responsible for the hypothermia observed in this patient.

In the laboratory findings, hyperglycemia and glycosuria are considered the hallmark of amitraz poisoning and are thought to be caused by inhibition of insulin release and stimulation of glucagon secretion [8]. Other laboratory findings are considered nonspecific and may include minimal increases in liver enzymes. Blood urea nitrogen, creatinine and electrolytes are usually not affected but polyuria has been reported in both children and adults and may be due to decreased antidiuretic hormone (ADH) and renin secretion, inhibition of $\mathrm{ADH}$ effect, and enhanced diuresis by increased glomerular filtration rate [2].

There is no known antidote for amitraz poisoning and therefore the management is largely supportive and symptomatic [9]. Supportive measures include airway management and oxygen and hemodynamic stabilization with intravenous fluids. Gastric lavage may be performed but it is recommended that this is done only after endotracheal intubation in order to avoid inhalation or aspiration pneumonitis from petroleum distillate in amitraz formulations [2]. Activated charcoal may be administered, severe bradycardia may respond to repeated doses of atropine and patients with respiratory depression may require mechanical ventilation [9]. Hyperglycemia, like in our patient, has been reported to resolve without any specific intervention. Neurological improvement usually occurs spontaneously within 6-28 hours of exposure [2].

Most patients with amitraz poisoning recover fully. However, a delay in diagnosis and/or delay in transfer to a hospital with facilities for mechanical ventilation could have fatal consequences. Death has most often been attributed to respiratory failure [1].

Pralidoxime (2-pyridine aldoxime methylchloride), the antidote used in organophosphate poisoning, has no role in the treatment of amitraz intoxication.

\section{CONCLUSION}

With the widespread use and easy availability of amitraz, it is likely that human intoxication with this agent will be on the rise. Possible amitraz poisoning should be considered in patients with a combination of pesticide exposure, hyperglycemia, and without the classical features of organophosphate poisoning. Increased awareness among clinicians is required for timely diagnosis and appropriate management of this potentially life-threatening intoxication.

$$
* * * * * * * * *
$$

\section{Acknowledgements}

I acknowledge the care and attention given to the patient by his family and all the medical and nursing staff of the pediatric ward in North Kinangop Catholic Hospital.

\section{Author Contributions}

Serah Kajuju Ngugi - Substantial contributions to conception and design, Acquisition of data, Analysis and interpretation of data, Drafting the article, Revising it critically for important intellectual content, Final approval of the version to be published

\section{Guarantor}

The corresponding author is the guarantor of submission.

\section{Conflict of Interest}

Authors declare no conflict of interest.

\section{Copyright}

(C) 2016 Serah Kajuju Ngugi. This article is distributed under the terms of Creative Commons Attribution License which permits unrestricted use, distribution and reproduction in any medium provided the original author(s) and original publisher are properly credited. Please see the copyright policy on the journal website for more information.

\section{REFERENCES}

1. Veale DJ, Wium CA, Muller GJ. Amitraz poisoning in South Africa: a two year survey (2008-2009). Clin Toxicol (Phila) 2011 Jan;49(1):40-4.

2. Yilmaz HL, Yildizdas DR. Amitraz poisoning, an emerging problem: epidemiology, clinical features, management, and preventive strategies. Arch Dis Child 2003 Feb;88(2):130-4.

3. Ertekin V, Alp H, Selimoglu MA, Karacan M. Amitraz poisoning in children: retrospective analysis of 21 cases. J Int Med Res 2002 Mar-Apr;30(2):203-5.

4. Agin H, Calkavur S, Uzun H, Bak M. Amitraz poisoning: clinical and laboratory findings. Indian Pediatr 2004 May;41(5):482-6.

5. Jones RD. Xylene/amitraz: a pharmacologic review and profile. Vet Hum Toxicol 1990 Oct;32(5):446-8. 
9. Eizadi-Mood N, Sabzghabaee AM, Gheshlaghi F, Yaraghi A. Amitraz poisoning treatment: still supportive? Iran J Pharm Res 2011 Winter;10(1):155-8.
Access full text article on other devices

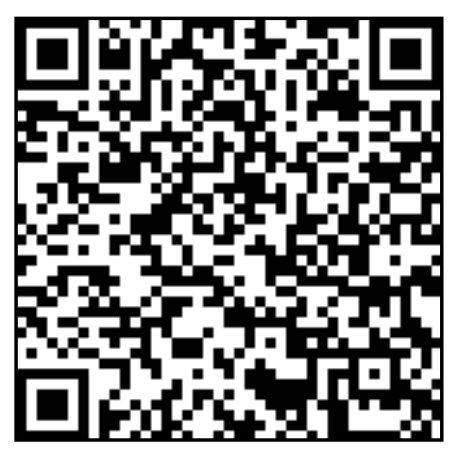

Access PDF of article on other devices

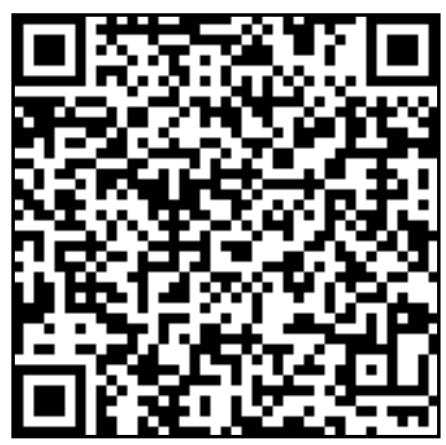

